IJMMS 31:5 (2002) 283-290

PII. S0161171202112245

http://ijmms.hindawi.com

(c) Hindawi Publishing Corp.

\title{
EXPONENTIAL INEQUALITIES FOR A CLASS OF OPERATORS
}

\section{HANS P. HEINIG}

Received 7 December 2001

Characterizations of weight pairs are proved for which weighted exponential-logarithmic integral inequalities are satisfied. The results given contain characterizations of weights for which inequalities involving the Riemann-Liouville and Laplace type operators hold.

2000 Mathematics Subject Classification: 26D10, 40A05.

1. Introduction. Consider the positive integral operator

$$
(K f)(x)=\int_{0}^{\infty} k(x, y) f(y) d y, \quad x \geq 0, f \geq 0, k \geq 0,
$$

where the kernel $k$ satisfies

$$
\begin{gathered}
k(\lambda x, \lambda y)=\lambda^{-1} k(x, y), \quad \lambda>0, \\
\int_{0}^{\infty} k(1, t) d t=1, \\
\exp \left(-\int_{0}^{\infty} k(1, t) \log t d t\right)=C_{0}<\infty, \\
k(t, x) \leq C_{1} \exp \left(\int_{0}^{\infty} k(x, y) \log k(t, y) d y\right), \\
s^{-1} k(s, x) \leq C_{2} \int_{0}^{\infty} y^{-1} k(y, s) k(y, x) d y,
\end{gathered}
$$

for some constants $C_{i}>0, i=0,1,2$, and all $s, t, x, y$. It is further assumed that the right-hand sides of (1.5) and (1.6) exist a.e. and are finite.

Let $\left(E_{k} f\right)(x)=\exp (K \log f)(x)$, then it is well known (cf. [1, 2, 3, 4], and the references therein) that for operators $K$ whose kernel satisfies (1.2), (1.3), and (1.4), the condition

$$
\sup _{y>0} \int_{0}^{\infty}\left[\frac{y}{x} k(x, y)\right] w(x) d x=C_{3}<\infty
$$

where $w(x)=u(x) \exp (K \log (1 / v))(x), u, v$ weight functions, implies that the inequality

$$
\int_{0}^{\infty} u(x)\left(E_{k} f\right)(x) d x \leq C_{4} \int_{0}^{\infty} v(x) f(x) d x
$$

is satisfied. 
For specific operators, largely of Hardy type, it was shown in [1, 3, 4] that (1.7) is also necessary for (1.8). However, in these studies, it was implicitly assumed that $E_{k} f$ (and $E_{u}(1 / v)$ ) exists a.e. and is measurable whenever $v f$ is integrable. Note, however, (cf. [2]) that $\log f(t)$ may be two signed, or it may be undefined whenever $f(t)=0$. Moreover, $E_{k} f$ may fail to exist even though $v f$ is integrable. For example, if $K$ is the Hardy averaging operator

$$
(K f)(x)=x^{-1} \int_{0}^{x} f(y) d y, \quad x \geq 0, f \geq 0,
$$

that is, $k(x, y)=x^{-1} \chi_{(0, x)}(y)$, then with $f(y)=e^{-1 / y},(K \ln f)(x)$ and hence $\left(E_{k} f\right)(x)$ fails to exist, although the right-hand side of (1.8) is finite if $v(x)=x^{-2}$.

These pathological situations may be avoided by a slight extension of the Lebesgue integral, so that in these cases $E_{k} f$ takes the value zero.

In this paper, we give conditions on $f$ (and $v$ ) which insures the measurability and existence a.e. of $E_{k} f$. Moreover, we give necessary and sufficient conditions on the weight functions $u$ and $v$-namely (1.7) -for which the exponential-logarithmic inequality (1.8) is satisfied. The novelty here is that (1.8) implies (1.7) for all operators $K$ whose kernel satisfy the easily verifiable conditions (1.2), (1.3), (1.4), (1.5), and (1.6). These main results are proved in the next section. In Section 3, we illustrate the main result by proving weight characterizations for the Riemann-Liouville operator with index not less than one, and a Laplace transform type operator. These results seem to be new.

As usual, we will take $\exp (-\infty)=0, \log 0=-\infty$, and expressions of the form $0 \cdot \infty$ are taken to be zero.

2. Main results. Following the arguments in [2], we define for real $x$

$$
|x|_{+}=\max \{0, x\}, \quad|x|_{-}=\max \{0,-x\}
$$

and agree that for the real measurable function $f(x)$, the (extended) Lebesgue integral

$$
\int f(x) d x=\int|f(x)|_{+} d x-\int|f(x)|_{-} d x
$$

exists, even if the second integral on the right is $+\infty$. In this case, the integral on the left-hand side has assigned value $-\infty$. The first integral on the right-hand side is not permitted to have value $+\infty$, so that under these conventions the integral exists if and only if $|f(x)|_{+}$is integrable.

In the next result, we establish the measurability and existence of $E_{k} f$ for general kernel $k$. The argument is similar to [2, Lemma 1] and is given here for completeness.

LEMmA 2.1. Assume that the kernel $k$ satisfies (1.2) and (1.3). If $f$ is a nonnegative measurable function, such that $|\log f(t)|_{+} / t$ is integrable on $(0, \infty)$, then $E_{k} f(x)$ exists a.e. in $[0, \infty)$ and is measurable.

Proof. For $t \in(-\infty, \infty)$, let $g(t)=\left|\log f\left(e^{t}\right)\right|_{+}$and $h(t)=e^{-t} k\left(1, e^{-t}\right)$. Then by hypotheses

$$
\int_{-\infty}^{\infty}|g(t)| d t=\int_{-\infty}^{\infty}\left|\log f\left(e^{t}\right)\right|_{+} d t=\int_{0}^{\infty}|\log f(y)|_{+} \frac{d y}{y}<\infty,
$$


where $y=e^{t}$, and by (1.3)

$$
\int_{-\infty}^{\infty}|h(t)| d t=\int_{-\infty}^{\infty} e^{-t} k\left(1, e^{-t}\right) d t=\int_{0}^{\infty} k(1, y) d y=1,
$$

where $e^{-t}=y$. Since both $h$ and $g$ are integrable on $(-\infty, \infty)$, its convolution exists a.e., that is,

$$
\begin{aligned}
\int_{-\infty}^{\infty} g(s-t) h(t) d t & =\int_{-\infty}^{\infty}\left|\log f\left(e^{s-t}\right)\right|_{+} e^{-t} k\left(1, e^{-t}\right) d t \\
& =\int_{0}^{\infty}\left|\log f\left(e^{s} y\right)\right|_{+} k(1, y) d y \\
& =\int_{0}^{\infty}|\log f(x y)|_{+} k(1, y) d y \\
& =\int_{0}^{\infty}|\log f(t)|_{+} k\left(1, \frac{t}{x}\right) \frac{d t}{x} \\
& =\int_{0}^{\infty} k(x, t)|\log f(t)|_{+} d t
\end{aligned}
$$

via obvious changes of variables and (1.2). Hence the last integral exists a.e. for $x \in(0, \infty)$ and is measurable. Thus

$$
(K \log f)(x)=\int_{0}^{\infty} k(x, t) \log f(t) d t \quad x>0
$$

exists a.e. with values in $[-\infty, \infty)$ and is measurable. Hence $E_{k} f(x)$ exists a.e. in $[0, \infty)$ as asserted.

REMARK 2.2. In the same way, we show that

$$
\int_{0}^{\infty} k(x, t) \log k(y, t) d t
$$

exists a.e. for fixed $x$ and $y$, and is measurable, whenever $|\log k(y, t)|_{+} / t$ is integrable. Only now we define

$$
g_{y}(t)=\left|\log k\left(y, e^{t}\right)\right|_{+}, \quad h_{x}(t)=e^{-t} k\left(x, e^{-t}\right), \quad t \in(-\infty, \infty) .
$$

The weight characterization for which (1.8) is satisfied is the following.

THEOREM 2.3. Suppose that $u$ and $v$ are positive measurable functions and that $|\log (1 / v(t))|_{+} / t$ is integrable. Let $w(x)=u(x) \exp (K \log (1 / v))(x)$, where $K$ is the operator (1.1) (with $k$ satisfying (1.2), (1.3), (1.4), (1.5), and (1.6)). If $f$ is nonnegative, measurable on $(0, \infty)$ such that $|\log f(t)|_{+} / t$ is integrable, then (1.8) is satisfied if and only if (1.7) holds. Moreover, if $C_{4}$ is the smallest constant satisfying (1.8), then $C_{3} /\left(C_{1} C_{2}\right) \leq C_{4} \leq C_{0} C_{3}$.

Proof. Since $t^{-1}|\log (1 / v)|_{+}$and $t^{-1}|\log f|_{+}$are integrable, $w(x)$ and $(K \log f)(x)$ exist a.e. by Lemma 2.1. Moreover, since

$$
\begin{aligned}
\int_{0}^{\infty} u(x) \exp (K \log f)(x) d x & =\int_{0}^{\infty} u(x) \exp \left[(K \log f v)+\left(K \log \frac{1}{v}\right)\right](x) d x \\
& =\int_{0}^{\infty} w(x) \exp (K \log f v)(x) d x
\end{aligned}
$$


and $(K \log f v)(x)$ exists a.e., (1.8) has the equivalent form

$$
\int_{0}^{\infty} w(x) \exp (K \log g)(x) d x \leq C_{4} \int_{0}^{\infty} g(x) d x
$$

on setting $g=v f$. Now by (1.2) and (1.4),

$$
\begin{aligned}
\int_{0}^{\infty} w & (x) \exp \left(\int_{0}^{\infty} k(x, y) \log g(y) d y\right) d x \\
& =\int_{0}^{\infty} w(x) \exp \left(\int_{0}^{\infty} k(x, x t)(\log g(x t)) x d t\right) d x \\
& =\int_{0}^{\infty} w(x) \exp \left(\int_{0}^{\infty} k(1, t)[\log (\operatorname{tg}(x t))-\log t] d t\right) d x \\
& =C_{0} \int_{0}^{\infty} w(x) \exp \left(\int_{0}^{\infty} k(1, t) \log (\operatorname{tg}(x t)) d t\right) d x \\
& \leq C_{0} \int_{0}^{\infty} w(x) \int_{0}^{\infty} k(1, t) \operatorname{tg}(x t) d t d x \\
& =C_{0} \int_{0}^{\infty} w(x) \int_{0}^{\infty} k\left(1, \frac{y}{x}\right) \frac{y}{x} g(y) \frac{d y}{x} d x \\
& =C_{0} \int_{0}^{\infty} g(y) \int_{0}^{\infty}\left[\frac{y}{x} k(x, y)\right] w(x) d x d y \\
\leq & C_{0} C_{3} \int_{0}^{\infty} g(y) d y,
\end{aligned}
$$

via obvious changes of variables, Jensen's inequality, and an interchange of order of integration. Thus, (2.10), or equivalently (1.8), holds with $C_{4} \geq C_{0} C_{3}$.

To prove the converse, define for fixed $t>0, g_{t}(x)=k(t, x)$. Since (1.5) is satisfied and the right-hand side of (1.5) exist, it follows that $\left(K \log g_{t}\right)(x)$ exists a.e. Also applying (1.2) and (1.3) shows that

$$
\int_{0}^{\infty} g_{t}(x) d x=\int_{0}^{\infty} k(t, x) d x=1 .
$$

Hence by (2.10) with $g$ replaced by $g_{t}$,

$$
\int_{0}^{\infty} w(x) \exp \left(K \log g_{t}\right)(x) d x \leq C_{4} .
$$

Applying (1.5) and (2.13) yields

$$
\begin{aligned}
(K w)(t) & =\int_{0}^{\infty} k(t, x) w(x) d x \\
& \leq C_{1} \int_{0}^{\infty} w(x)\left(\exp \int_{0}^{\infty} k(x, y) \log k(t, y) d y\right) d x \\
& =C_{1} \int_{0}^{\infty} w(x) \exp \left(K \log g_{t}\right)(x) d x \leq C_{1} C_{4} .
\end{aligned}
$$

Now define $K_{1}$ by

$$
\left(K_{1} h\right)(x)=x \int_{0}^{\infty} y^{-1} k(y, x) h(y) d y,
$$


then by (1.7) the result follows if $\left(K_{1} w\right)(x) \leq C$ for some constant $C$ and all $x>0$. But by (1.2) and (1.3),

$$
\left(K_{1} 1\right)(x)=\int_{0}^{\infty} k(y, x) \frac{x}{y} d y=\int_{0}^{\infty} k\left(1, \frac{x}{y}\right) x y^{-2} d y=\int_{0}^{\infty} k(1, s) d s=1
$$

with $s=x / y$, and since we have seen that $(K w)(t) \leq C_{1} C_{4}$, it suffices to show that

$$
\left(K_{1} w\right)(x) \leq C^{1}\left(K_{1}(K w)\right)(x)
$$

is satisfied for some $C^{1}$. For then, $\left(K_{1} w\right)(x) \leq C^{1} C_{1} C_{4}$. But by (1.6)

$$
\begin{aligned}
\left(K_{1}(K w)\right)(x) & =x \int_{0}^{\infty} k(y, x) y^{-1}(K w)(y) d y \\
& =x \int_{0}^{\infty} y^{-1} k(y, x) \int_{0}^{\infty} k(y, s) w(s) d s d y \\
& =\int_{0}^{\infty} w(s)\left[x \int_{0}^{\infty} y^{-1} k(y, x) k(y, s) d y\right] d s \\
& \geq \frac{1}{C_{2}} \int_{0}^{\infty} w(s) \frac{x}{s} k(s, x) d s=\frac{1}{C_{2}}\left(K_{1} w\right)(x),
\end{aligned}
$$

so that (2.17) is satisfied with $C^{1}=C_{2}$. Hence (1.7) is satisfied with $C_{3} \leq C_{1} C_{2} C_{4}$, and the result follows.

3. Applications. If $f$ and $v$ satisfy the conditions of Theorem 2.3 and $P_{\beta}$ is the operator defined by

$$
\left(P_{\beta} f\right)(x)=\beta x^{-\beta} \int_{0}^{x} t^{\beta-1} f(t) d t, \quad \beta>0, x>0,
$$

then

$$
\int_{0}^{\infty} u(x) \exp \left(P_{\beta} \log f\right)(x) d x \leq C \int_{0}^{\infty} v(x) f(x) d x
$$

if and only if

$$
\sup _{y>0} \int_{y}^{\infty}\left[\frac{y^{\beta}}{x^{\beta+1}}\right] w(x) d x<\infty,
$$

where $w(x)=u(x) \exp \left[P_{\beta} \log (1 / v)\right](x)$.

This follows from Theorem 2.3 provided that $k(x, y)=\beta x^{-\beta} y^{\beta-1} \chi_{(0, x)}(y)$ satisfies conditions (1.2), (1.3), (1.4), (1.5), and (1.6). However, straightforward calculations show that these conditions are indeed satisfied. Since this result follows also from [1, Theorem 2.2], we omit the details.

The next application concerns the Riemann-Liouville operator

$$
\left(I_{\alpha} f\right)(x)=\frac{\alpha}{x^{\alpha}} \int_{0}^{x}(x-y)^{\alpha-1} f(y) d y, \quad \alpha \geq 1, x>0,
$$


COROLlary 3.1. Suppose that $u$ and $v$ are positive functions and $t^{-1}|\log (1 / v(t))|_{+}$ is integrable. If $f$ is nonnegative, measurable, and $t^{-1}|\log f(t)|_{+}$is integrable, then

$$
\int_{0}^{\infty} u(x) \exp \left(I_{\alpha} \log f\right)(x) d x \leq C \int_{0}^{\infty} v(x) f(x) d x
$$

is satisfied if and only if

$$
\sup _{y>0} \alpha y \int_{y}^{\infty} x^{-\alpha-1}(x-y)^{\alpha-1} w(x) d x=C_{3}<\infty
$$

where $w(x)=u(x) \exp \left(I_{\alpha} \log (1 / v)\right)(x)$.

Proof. We need to show that the kernel $k(x, y)=\alpha / x^{\alpha}(x-y)^{\alpha-1} \chi_{(0, x)}(y)$ satisfies (1.2), (1.3), (1.4), (1.5), and (1.6), for then the result follows from Theorem 2.3. Clearly (1.2) and (1.3) are satisfied and since

$$
\int_{0}^{\infty} k(1, t) \log (t) d t=\alpha \int_{0}^{1}(1-t)^{\alpha-1} \log t d t, \quad \alpha \geq 1,
$$

converges, (1.4) also holds. To show that (1.5) is satisfied, we must show that for some $C_{1}>0$

$$
\frac{\alpha}{t^{\alpha}}(t-x)^{\alpha-1} \chi_{(0, t)}(x) \leq C_{1} \exp \int_{0}^{x} \frac{\alpha}{x^{\alpha}}(x-y)^{\alpha-1} \log \left(\frac{\alpha}{t^{\alpha}}(t-y)^{\alpha-1} \chi_{(0, t)}(y)\right) d y
$$

holds. If $y \geq t, \log \left(\left(\alpha / t^{\alpha}\right)(t-y)^{\alpha-1} \chi_{(0, t)}(y)\right)=-\infty$, so the right-hand side of (3.8) is zero. But since $y \leq x$, it follows that $t \leq x$ so the left-hand side of (3.8) is also zero and the inequality holds in this case trivially. Now if $0<y<t$, then the right-hand side of (3.8) is

$$
\begin{aligned}
C_{1} \exp & \left\{\frac{\alpha}{x^{\alpha}} \int_{0}^{x}(x-y)^{\alpha-1}\left[\log \frac{\alpha}{t^{\alpha}}+\log (t-y)^{\alpha-1}\right] d y\right\} \\
& =C_{1} \exp \left[\frac{\alpha}{x^{\alpha}}\left(\log \frac{\alpha}{t^{\alpha}}\right) \frac{x^{\alpha}}{\alpha}\right] \exp \frac{\alpha(\alpha-1)}{x^{\alpha}} \int_{0}^{x}(x-y)^{\alpha-1} \log (t-y) d y \\
& =\frac{C_{1} \alpha}{t^{\alpha}} \exp \frac{\alpha(\alpha-1)}{x^{\alpha}} J
\end{aligned}
$$

where

$$
\begin{aligned}
J & =\int_{0}^{x}(x-y)^{\alpha-1} \log (t-y) d y=\frac{x^{\alpha}}{\alpha} \log (t)-\frac{1}{\alpha} \int_{0}^{x}(x-y)^{\alpha} \frac{1}{t-y} d y \\
& \geq \frac{x^{\alpha}}{\alpha} \log t-\frac{x^{\alpha}}{\alpha} \int_{0}^{x} \frac{d y}{t-y}=\frac{x^{\alpha}}{\alpha} \log t+\frac{x^{\alpha}}{\alpha}[\log (t-x)-\log t] \\
& =\frac{x^{\alpha}}{\alpha} \log (t-x) .
\end{aligned}
$$

Substituting into (3.9) shows that (3.9) is not smaller than

$$
\frac{C_{1} \alpha}{t^{\alpha}} \exp \frac{\alpha(\alpha-1)}{x^{\alpha}}\left(\frac{x^{\alpha}}{\alpha} \log (t-x)\right)=\frac{C_{1} \alpha}{t^{\alpha}}(t-x)^{\alpha-1}
$$

so that (3.8) and hence (1.5) is satisfied with $C_{1}=1$. 
Finally, we will show that (1.6) holds, that is, for some $C_{2}>0$

$$
\begin{aligned}
\frac{\alpha}{x^{\alpha+1}}(s & -x)^{\alpha-1} \chi_{(0, s)}(x) \\
& \leq C_{2} \int_{0}^{\infty} \frac{\alpha}{y^{\alpha}}(y-s)^{\alpha-1} \chi_{(0, y)}(s) \frac{\alpha}{y^{\alpha}}(y-x)^{\alpha-1} \chi_{(0, y)}(x) \frac{d y}{y}
\end{aligned}
$$

is satisfied. But since $\chi_{(0, y)}(s)=\chi_{(s, \infty)}(y), \chi_{(0, y)}(x)=\chi_{(x, \infty)}(y)$, and $\chi_{(s, \infty)}(y) \chi_{(x, \infty)}(y)$ $=\chi_{(\max (s, x), \infty)}(y)$, the term on the right-hand side in (3.12) is

$$
C_{2} \alpha^{2} \int_{0}^{\infty} y^{-2 \alpha-1}(y-s)^{\alpha-1}(y-x)^{\alpha-1} \chi_{(\max (s, x), \infty)}(y) d y .
$$

If $x<s$ this is

$$
\begin{aligned}
C_{2} \alpha^{2} \int_{s}^{\infty} & y^{-2 \alpha-1}(y-s)^{\alpha-1}(y-x)^{\alpha-1} d y \\
& \geq C_{2} \alpha^{2}(s-x)^{\alpha-1} \int_{s}^{\infty} y^{-2 \alpha-1}(y-s)^{\alpha-1} d y \\
& =C_{2} \alpha^{2}(s-x)^{\alpha-1} s^{-\alpha-1} \int_{1}^{\infty} t^{-2 \alpha-1}(t-1)^{\alpha-1} d t \quad(y=s t),
\end{aligned}
$$

so that (3.12) and hence (1.6) holds with $C_{2}=\left(\alpha \int_{1}^{\infty} t^{-2 \alpha-1}(t-1)^{\alpha-1} d t\right)^{-1}$. If $x \geq s$, (3.12) holds trivially. This proves the corollary.

In the final result, we consider the operator

$$
\left(L_{a} f\right)(x)=\frac{1}{x \Gamma(1+1 / a)} \int_{0}^{\infty} e^{-(y / x)^{a}} f(y) d y, \quad a \geq 1, x \geq 0,
$$

where $\Gamma$ is the Gamma function.

COROLLARY 3.2. Suppose that $u, v, w$, and $f$ satisfy the conditions of Corollary 3.1. Then

$$
\int_{0}^{\infty} u(x) \exp \left(L_{a} \log f\right)(x) d x \leq C \int_{0}^{\infty} v(x) f(x) d x, \quad a \geq 1,
$$

is satisfied if and only if

$$
\sup _{y>0} \frac{y}{\Gamma(1+1 / a)} \int_{0}^{\infty} \frac{e^{-(y / x)^{a}}}{x^{2}} w(x) d x<\infty .
$$

Proof. The result follows from Theorem 2.3 if one shows that the kernel

$$
k(x, y)=\frac{1}{x \Gamma(1+1 / a)} e^{-(y / x)^{a}}
$$

satisfies conditions (1.2), (1.3), (1.4), (1.5), and (1.6). Clearly, (1.2) is satisfied and so is (1.3) since by definition of the Gamma function

$$
\begin{aligned}
\int_{0}^{\infty} k(1, y) d y & =\frac{1}{\Gamma(1+1 / a)} \int_{0}^{\infty} e^{-y^{a}} d y \\
& =\frac{1}{a \Gamma(1+1 / a)} \int_{0}^{\infty} e^{-t} t^{1 / a-1} d t \\
& =\frac{(1 / a) \Gamma(1 / a)}{\Gamma(1+1 / a)} \\
& =1 .
\end{aligned}
$$


Moreover, since

$$
\int_{0}^{\infty} k(1, t) \log t d t=\frac{1}{\Gamma(1+1 / a)} \int_{0}^{\infty} e^{-t^{a}} \log t d t
$$

converges, then (1.4) holds.

To show that (1.5) is satisfied, we must show that for some $C_{1}>0$

$$
\frac{1}{t \Gamma(1+1 / a)} e^{-(x / t)^{a}} \leq C_{1} \exp \left(\int_{0}^{\infty} \frac{e^{-(y / x)^{a}}}{x \Gamma(1+1 / a)} \log \left(\frac{e^{-(y / t)^{a}}}{t \Gamma(1+1 / a)}\right) d y\right)
$$

holds. But the integral on the right-hand side is

$$
\begin{gathered}
\frac{1}{x \Gamma(1+1 / a)}\left[\log \left(\frac{1}{t \Gamma(1+1 / a)}\right) \int_{0}^{\infty} e^{-(y / x)^{a}} d y-\int_{0}^{\infty} e^{-(y / x)^{a}}\left(\frac{y}{t}\right)^{a} d y\right] \\
=\frac{1}{x \Gamma(1+1 / a)}\left[\log \left(\frac{1}{t \Gamma(1+1 / a)}\right) x \Gamma\left(1+\frac{1}{a}\right)-\frac{x^{a+1}}{t^{a} a} \Gamma\left(1+\frac{1}{a}\right)\right] \\
=\log \left(\frac{1}{t \Gamma(1+1 / a)}\right)-\left(\frac{x}{t}\right)^{a} \cdot \frac{1}{a} \geq \log \left(\frac{1}{t \Gamma(1+1 / a)}\right)-\left(\frac{x}{t}\right)^{a}
\end{gathered}
$$

and taking exponents, we obtain (3.21) with $C_{1}=1$.

Finally,

$$
\begin{aligned}
\int_{0}^{\infty} y^{-1} k(y, s) k(y, x) d y & \geq \frac{1}{[\Gamma(1+1 / a)]^{2}} \int_{s}^{\infty} y^{-3} e^{-(s / y)^{2}-(x / y)^{2}} d y \\
& \geq \frac{e^{-1} e^{-(x / s)^{a}}}{[\Gamma(1+1 / a)]^{2}} \int_{s}^{\infty} y^{-3} d y=\frac{e^{-1}}{2 \Gamma(1+1 / a)} \frac{k(s, x)}{s},
\end{aligned}
$$

so that (1.6) is also satisfied. This proves the result.

Note that for $a=1$, Corollary 3.2 is [1, Theorem 2.6].

\section{REFERENCES}

[1] H. P. Heinig, R. Kerman, and M. Krbec, Weighted exponential inequalities, Georgian Math. J. 8 (2001), no. 1, 69-86.

[2] E. R. Love, Inequalities related to Knopp's inequality, J. Math. Anal. Appl. 137 (1989), no. 1, 173-180.

[3] B. Opic and P. Gurka, Weighted inequalities for geometric means, Proc. Amer. Math. Soc. 120 (1994), no. 3, 771-779.

[4] L. Pick and B. Opic, On the geometric mean operator, J. Math. Anal. Appl. 183 (1994), no. 3, 652-662.

Hans P. Heinig: Department of Mathematics and STAtistics, McMaster University, HAMILTON, ONTARIO, CANADA L8S 4K1

E-mail address: heinig@mcmai 1 .cis.mcmaster.ca 


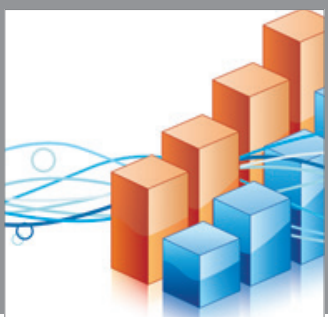

Advances in

Operations Research

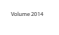

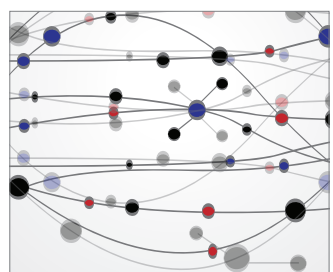

\section{The Scientific} World Journal
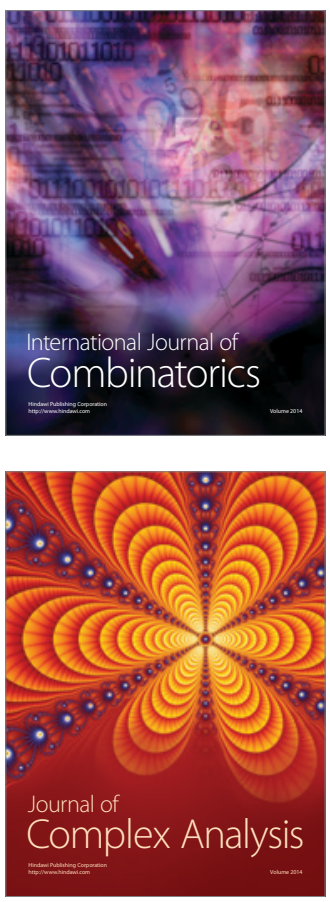

International Journal of

Mathematics and

Mathematical

Sciences
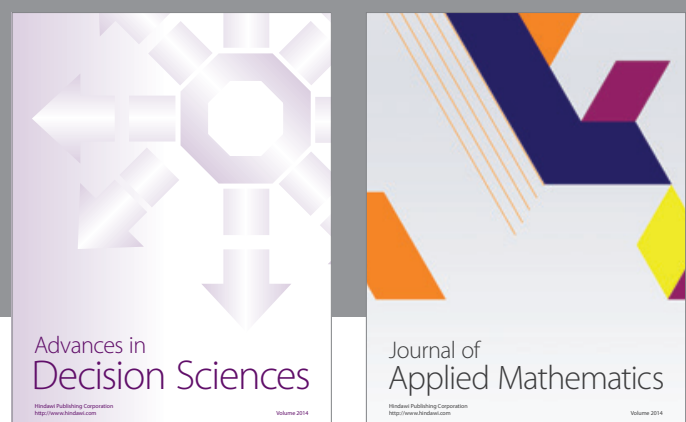

Journal of

Applied Mathematics
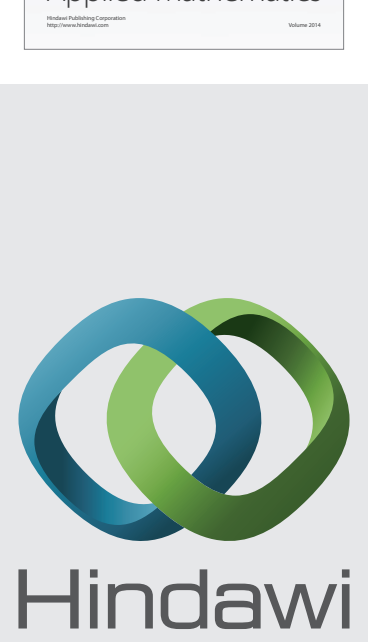

Submit your manuscripts at http://www.hindawi.com
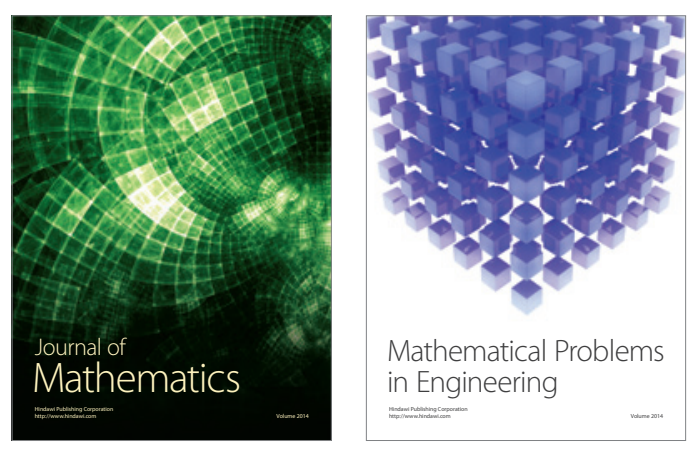

Mathematical Problems in Engineering
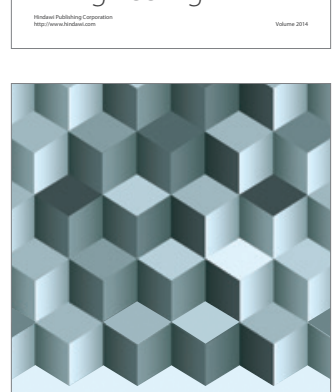

Journal of

Function Spaces
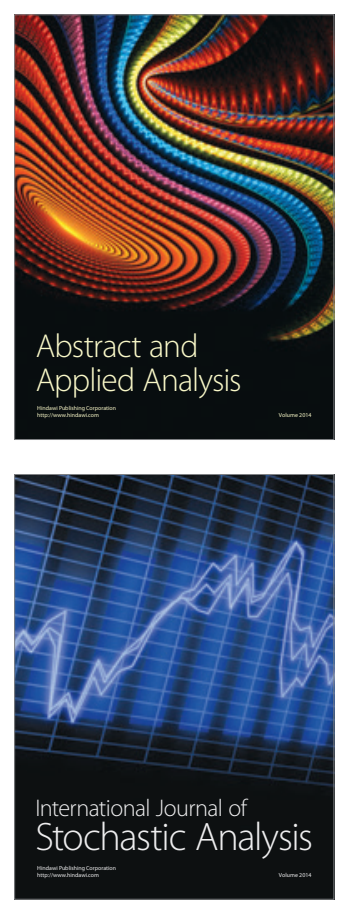

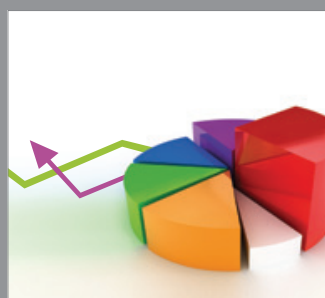

ournal of

Probability and Statistics

Promensencen
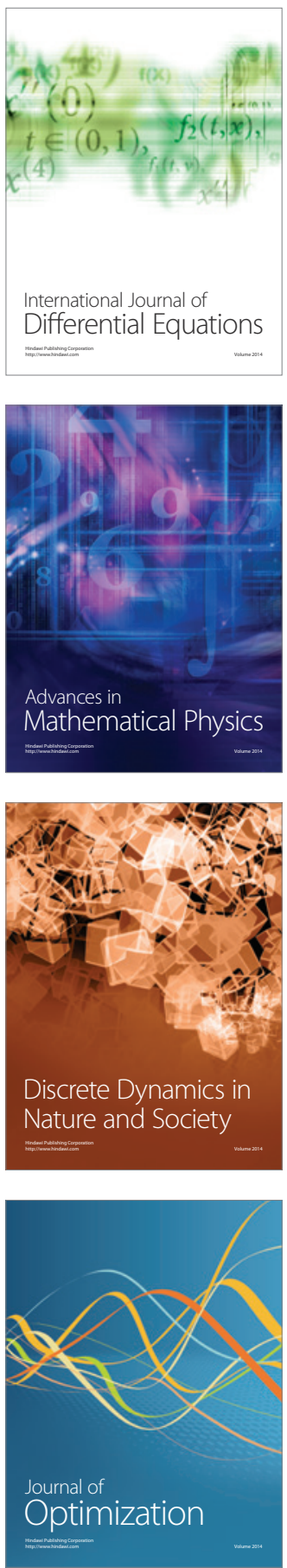